\title{
Note de correction: Le théâtre, une méthode pédagogique au profil du changement de comportement dans les soins : revue systématique de la littérature
}

\section{Educ Ther Patient/Ther Patient Educ 2019, 11, 20201, https://doi.org/10.1051/tpe/ 2019009}

Simon-Decap Mabakutuvangilanga-Ntela ${ }^{1,2, *}$, Daniel Ishoso Katwashi ${ }^{3,4}$, Mathieu Ahouah ${ }^{1}$, Félicien Tshimungu Kandolo ${ }^{2}$, Monique Rothan Tondeur ${ }^{1,5}$ et Aurore Margat ${ }^{1}$

${ }^{1}$ Université Paris 13, Sorbonne Paris Cité, Chaire Recherche Sciences Infirmières, Laboratoire Éducations et Pratiques de Santé (LEPSEA 3412), UFR SMBH, 93017 Bobigny, France

${ }^{2}$ Section des sciences Infirmières, Institut Supérieur des Techniques de Médicales de Kinshasa, Kinshasa, République Démocratique du Congo

${ }^{3}$ École de santé-Publique, Université de Kinshasa, Département de santé communautaire, Kinshasa, République Démocratique du Congo

${ }^{4}$ Centre de recherche «Politiques et systèmes de santé - Santé internationale», École de santé publique de l'Université libre de Bruxelles (ULB), Bruxelles, Belgique

5 AP-HP, Chaire Recherche Sciences Infirmières, Paris, France

Reçu le 8 juillet 2019 / Accepté le 8 juillet 2019

Une erreur s'est produite dans le résumé de la version PDF de l'article «Le théâtre, une méthode pédagogique au profil du changement de comportement dans les soins : revue systématique de la littérature » publié dans le volume 11, 20201 (2019). La première phrase était incomplète, la version actuelle a été modifiée. L'éditeur s'excuse pour cette erreur.

\footnotetext{
* Auteur de correspondance : decapntela@gmail.com
} 